\title{
Late Diagnosis of Progressive Pseudorheumatoid Dysplasia in an Adolescent Patient: A Case Report and Review of the Literature
}

\author{
(1) Maria A Karalexi1, (1) Anna Papadopoulou', (1) Olympia Papakonstantinou², (1) Marina Mitrogiorgou1,

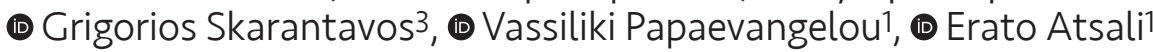

\begin{abstract}
${ }^{1}$ National and Kapodistrian University of Athens, Attikon University Hospital, Clinic of Pediatrics, Athens, Greece ${ }^{2}$ National and Kapodistrian University of Athens, Attikon University Hospital, Clinic of Radiology, Athens, Greece ${ }^{3}$ National and Kapodistrian University of Athens, Attikon University Hospital, Clinic of Rheumatology and Bone Unit, Division of Orthopedic, Athens, Greece
\end{abstract}

\begin{abstract}
We present an adolescent girl with severe kyphoscoliosis and inability of walking due to progressive pseudorheumatoid dysplasia (PPD) who was initially misdiagnosed as juvenile idiopathic arthritis for 9 years. The lack of inflammatory joint involvement and inflammatory laboratory parameters, along with the characteristic radiological findings should raise suspicion for PPD.

Keywords: Progressive pseudorheumatoid dysplasia, childhood, late diagnosis, juvenile idiopathic arthritis, intensive rehabilitation treatment
\end{abstract}

\section{Introduction}

Progressive pseudorheumatoid dysplasia (PPD), known as spondyloepiphyseal dysplasia tarda with progressive arthropathy, is a rare genetic bone disorder characterized by the progressive degeneration of articular cartilage affecting both the axial skeleton and peripheral joints (1). PPD occurs due to loss-of-function mutations in the WNT1-inducible signaling protein 3 gene, currently known as Connective tissue growth factor, Cysteine-rich 61, Nephroblastoma over-expressed (CCN6) gene (2). The prevalence of PPD is estimated at one per million children with a slight predominance among females and consanguineous populations (1).
The mean age ranges between 3 and 8 years (3). PPD is characterized by polyarticular involvement, gait abnormalities and fatigability. Symptoms may clinically mimic those of juvenile idiopathic arthritis (JIA) resulting in a late diagnosis. The absence of inflammatory joint involvement and raised inflammatory laboratory parameters, characteristic radiological findings (enlarged epiphyses, platyspondyly), and poor response to antiinflammatory/immunosuppressive treatment should raise suspicion for PPD (4). Due to the rarity of the disease, to-date literature is limited (approximately 64 studies) among which 28 are case reports and 27 are case series with less than 10 patients (1,5-7); even fewer are the large series counting more than 30 PPD patients $(1,8)$. 
This is the first report of a PPD patient in Greece, an adolescent girl who was misdiagnosed and treated as IIA for 9 years.

\section{Case presentation}

A 13.5-year-old girl presented to our department due to severe pain in both hips and gradual difficulty of walking. Familiar history was uneventful for rheumatic and autoimmune diseases. Parents were not consanguineous and the child had normal facial appearance and intelligence. Since the age of 4 years, the girl presented pain and progressive stiffness and swelling of small joints of hands and both ankles. She was then diagnosed as suffering from seronegative polyarticular $\|$ A. Treatment with corticosteroids (5 $\mathrm{mg}$ prednisolone/day), methotrexate (10 mg/week) and folic acid (5 mg/week) was initiated. Due to poor response, treatment was scaled up including subcutaneous etanercept (25 mg/week) one year later; however, etanercept had also minimal clinical impact. Despite treatment, the patient gradually presented back pain. Radiology of the spine showed kyphoscoliosis and platyspondyly, which was not taken into account at that time. Consequently, during 9 years the girl remained under disease-modifying antirheumatic drugs (DMARD) and antiTNF treatment with no improvement, instead worsening of her symptoms resulting in progressive emergence of difficulty of walking.
Upon examination at our department, the girl presented on wheelchair, unable to walk on her own. Her weight and height were short for age $\left(<3^{\text {rd }}\right.$ percentile). She had severe kyphoscoliosis of thoracic spine and restricted mobility of cervical spine and coxofemoral joints. There was enlargement and contractures of almost all peripheral joints, i.e. elbows, wrists, metacarpophalangeal, distal and proximal interphalangeal joints of both hands and ankles, resulting in severe restriction of their movement (Figures $1 a, b, c)$. Enlargement in peripheral joints was of bony consistence resulting in camptodactyly, while no active arthritis was detected.

Laboratory investigation showed normal white blood cell count, erythrocyte sedimentation rate (ESR), C-reactive protein (CRP), rheumatoid factors, antinuclear and anticyclic citrullinated peptide antibodies. Ophthalmologic examination was normal. Review of the child's medical history showed no signs of raised CRP/ESR since the beginning of the disease. Radiograph of the hands revealed diffuse osteopenia, widening of the metacarpal and phalangeal epiphyses and loss of joint space without erosive lesions (Figure 1d). Radiology of the hips and spine showed degenerative changes with almost complete destruction of hip joints and platyspondyly (Figures $1 e, f, g$ ). No soft tissue swelling or erosions could be seen. Peripheral quantitative computed tomography (pQCT; slices 4, 14 and 38\% of tibia

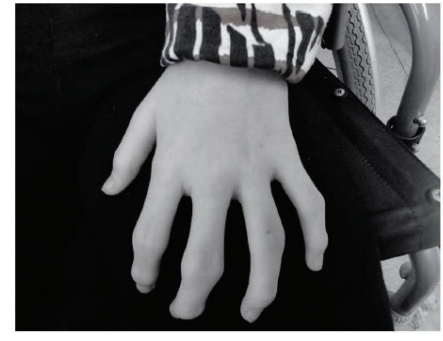

a

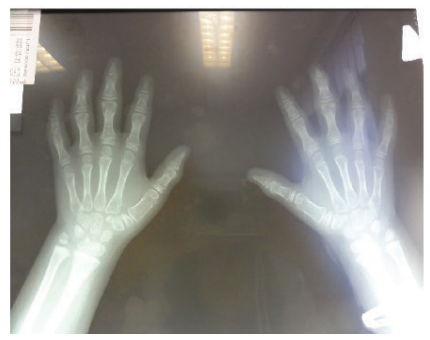

d

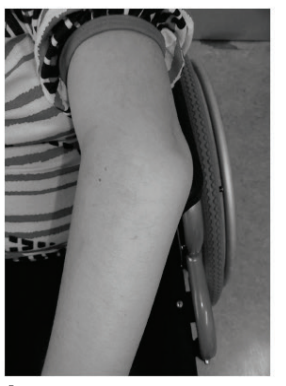

b

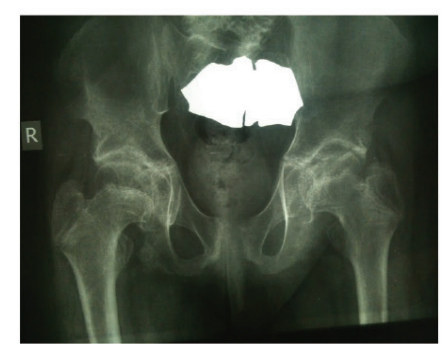

e

c
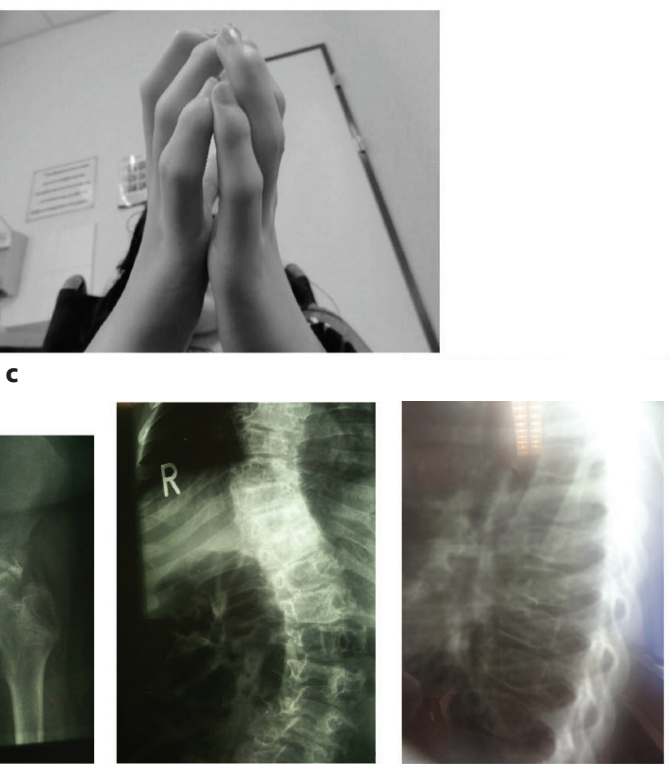

Figure 1. Patient 13 years old: Enlargement and contractures of metacarpophalangeal and interphalangeal joints (a) with limitation of extension of the fingers (b); and enlargement and contractures of the elbow (c). Hand X-rays showing osteopenia, enlargement of the metaphyses and epiphyses at the metacarpals and phalanges, with loss of joint space, without erosions (d). Coxofemoral X-rays showing severe degenerative changes (e) and spine X-rays showing severe scoliosis ( $f$ ) and flattened thoracic and lumbar vertebral bodies with irregularities of their surfaces (platyspondyly) ( $g$ ) 
length) showed extremely reduced trabecular volumetric density $\left(86 \mathrm{mg} / \mathrm{cm}^{3}\right)$, cortical volumetric density and derived bone strength (slice $38 \%-344 \mathrm{~mm}^{3}$ ).

The aforementioned findings raised suspicion for PPD. Direct sequencing of all coding genes and their flanking regions of the CCN6 gene revealed the c.156C>A (p.Cys52*), and the c.248G $>$ A (p.Gly83Glu) mutations both located in exon 2, confirming the diagnosis of PPD (Figure 2).

Intensive rehabilitation treatment was initiated. One year later, the patient started walking with crutches and the mobility of almost all joints and the parameters of PQCT were significantly improved. Currently, the girl is able to walk on her own. Given that the range of motion of her hips remains minimal (inability to climb stairs and dress alone) she has been referred to orthopedic surgeons and a totalhip replacement arthroplasty is being planned.

The proxies of the patient have signed a consent to publication form and the signed form is held by the treating institution.

\section{Discussion}

PPD is a progressive autosomal recessive disorder which caused decreased joint mobility, osseous swelling of the interphalangeal and other joints, and platyspondyly (1).

The disease is due to biallelic mutations in the CCN6 gene located on chromosome $6 \mathrm{q} 22$ (2). This gene encodes a 354-amino acid protein, a member of the connective tissue growth factors CCN gene family. The CCN6 protein is expressed in skeletal-derived cells and participates in angiogenesis, maintenance of cartilage integrity and bone growth. Seventy-six CCN6 gene mutations have been reported so far, the majority of which located in exon 2 $(1,2)$. In our case, we identified two mutations, the most frequent pathogenic mutation c.156C $>$ A (p.Cys52*), and the c.248G>A (p.Gly83Glu) both located in exon 2; these mutations have been described before in children with PPD (9).

Clinically, patients appear normal at birth, have subtle symptoms by the age of 3 years and develop later a progressive degenerative joint disease characterized by enlarged metacarpophalangeal and interphalangeal joints, kyphoscoliosis, short stature and severe reduction in almost all joints' mobility with multiple contractures (Table I) (6). The characteristic radiologic findings include platyspondyly, large femoral and tibial epiphyses, enlarged epi-metaphyseal portions of the metacarpals and phalangs, and narrow joint spaces $(1,4)$. Cervical spine involvement seems to be a significant prognostic factor due to severe neurological manifestations that may be caused by spinal canal stenosis (1).

The diagnosis of PPD is challenging with many children initially misdiagnosed as IIA $(4,10)$. The differential diagnosis also includes myopathy, camptodactyly-arthropathycoxa-vara-pericarditis syndrome and some types of mucopolysaccharidosis. Despite the onset of first symptoms

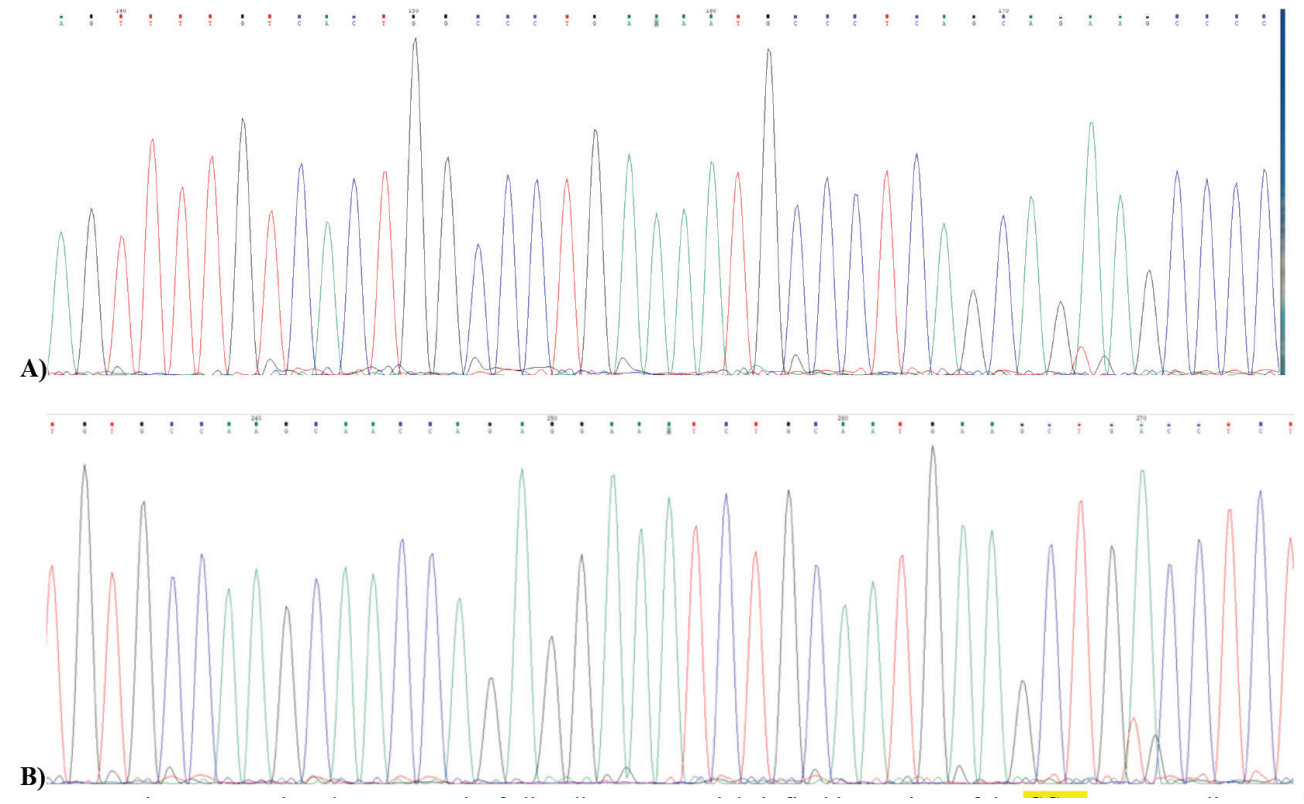

Figure 2. Direct sequencing chromatograph of all coding genes and their flanking regions of the CCN6 gene revealing (A) the c.156C>A (p.Cys52*) and (B) the c.248G $>$ A (p.Gly83Glu) mutations in the patient 
Table I. Main clinical, laboratory and radiologic features of progressive pseudorheumatoid dysplasia (PPD) compared to juvenile idiopathic arthritis

\begin{tabular}{|c|c|c|c|}
\hline Clinical, laboratory and radiologic features & PPD & JIA & Patient's features \\
\hline Mean age at onset (years) & $3-8$ & $1-3$ and $8-10$ & 4 \\
\hline $\begin{array}{l}\text { Familiar history (relatives with similar symptoms, consanguineous } \\
\text { marriage) }\end{array}$ & + & uncommon & - \\
\hline Joint contractures & + & + & + \\
\hline Inflammatory joint involvement & - & + & - \\
\hline Extra-skeletal clinical manifestations & - & + & - \\
\hline Inflammatory laboratory markers & - & + & - \\
\hline \multicolumn{4}{|l|}{ Radiological findings } \\
\hline Soft tissue swelling & - & + & - \\
\hline Bony erosions & - & + & - \\
\hline Narrow articular space & + & + & + \\
\hline Ankylosis & + & + & + \\
\hline $\begin{array}{l}\text { Narrowed joint space, epiphyseal dysplasia, enlarged epiphyses and } \\
\text { irregular acetabulum }\end{array}$ & + & - & + \\
\hline Platyspondyly & + & - & + \\
\hline Kyphoscoliosis & + & $+/-$ & + \\
\hline Anterior ossification defects in vertebrae & + & - & - \\
\hline Spondyloepiphyseal dysplasia & + & - & + \\
\hline Response to anti-rheumatic/anti-inflammatory treatment & poor & + & no \\
\hline
\end{tabular}

in early childhood, the diagnosis of PPD is usually delayed up to the second decade of life, and patients often receive unnecessary anti-inflammatory and immunosuppressive treatments (6). Although no diagnostic criteria have been established, "typical PPD" is defined as the presence of early-onset stiffness and pain in multiple joints, enlarged interphalangeal joints, normal inflammatory parameters, and the absence of extra-skeletal manifestations (Table I) (1). It is thus critical to reevaluate the clinical clues in cases without recovery or good response to treatment, as the patient described herein, and discuss such cases in multidisciplinary councils. Moreover, a lateral radiograph of the spine or a full skeletal survey could direct towards the diagnosis, which is thereafter usually confirmed by molecular genetic testing $(9,10)$. However, genetic testing of genomic DNA extracted from blood leucocytes may be negative given that intronic mutations in CCN6 can only be detected by analyzing mRNA from cultured skin fibroblasts (11). Thus, a skin biopsy could be a useful diagnostic tool in cases with suspicion of PPD and negative mutation screening of genomic DNA (1). Genetic testing is recommended for family counselling (3).

The treatment of PPD remains supportive. The poor response to DMARD is typical of PPD, whereas antiinflammatory treatment has also minimal clinical impact (6). Early-onset rehabilitation therapy is of utmost importance to preserve joint mobility. Surgical interventions include hip and knee joint replacement, realignment of the lower limbs and spinal surgery (4). The best timing for joint replacement depends on the patient's condition, but it is not recommended before epiphyseal closure to avoid leg length discrepancy, namely before the ages 12-18 for girls (usually 15-16 years) and 14-19 for boys (usually 18-19 years) (1).

PPD is a non-inflammatory skeletal disorder clinically simulating IA. Increased awareness and timely recognition of this disease could spare children of unnecessary treatments and offer access to family genetic counselling. Larger studies could refine our understanding of the pathogenesis of PPD allowing hopefully the development of novel etiological treatments. 


\section{Ethics}

Informed Consent: The proxies of the patient have signed a consent to publication form and the signed form is held by the treating institution.

Peer-review: Externally and internally peer-reviewed.

\section{Authorship Contributions}

Concept: E.A., Design: E.A., Analysis or Interpretation: A.P., M.M., Data Collection or Processing: A.P., Critical Review: M.A.K., A.P., O.P., M.M., G.S., V.P., E.A.

Conflict of Interest: No conflict of interest was declared by the authors.

Financial Disclosure: The authors declared that this study received no financial support.

\section{References}

1. Torreggiani S, Torcoletti M, Campos-Xavier B, et al. Progressive pseudorheumatoid dysplasia: a rare childhood disease. Rheumatol Int 2019; 39:441-52.

2. Pode-Shakked B, Vivante A, Barel O, et al. Progressive pseudorheumatoid dysplasia resolved by whole exome sequencing: a novel mutation in WISP3 and review of the literature. BMC Med Genet 2019; 20:53.

3. Alawbathani S, Kawalia A, Karakaya M, Altmuller ), Nurnberg P, Cirak S. Late diagnosis of a truncating WISP3 mutation entails a severe phenotype of progressive pseudorheumatoid dysplasia. Cold Spring Harb Mol Case Stud 2018; 4:a002139. doi: 10.1101/ mcs.a002139.
4. Yildizgoren MT, Sahin A, Osmanoglu K, Turhanoglu AD. Progressive pseudorheumatoid dysplasia with delayed Diagnosis. J Clin Rheumatol 2015; 21:329.

5. Bhavani CSL, Shah H, Shukla A, Dalal A, Girisha KM. Progressive Pseudorheumatoid Dysplasia. In: Adam MP, Ardinger HH, Pagon RA, et al., (eds). Seattle, GeneReviews, 1993.

6. Ekbote $A V$, Danda D, Kumar S, Danda S, Madhuri $V$, Gibikote S. A descriptive analysis of 14 cases of progressivepsuedorheumatoid-arthropathy of childhood from south India: review of literature in comparison with juvenile idiopathic arthritis. Semin Arthritis Rheum 2013; 42:582-9.

7. Wang Y, Xiao K, Yang Y, et al. CCN6 mutation detection in Chinese patients with progressive pseudo-rheumatoid dysplasia and identification of four novel mutations. Mol Genet Genomic Med 2020; 8:e1261. doi: 10.1002/mgg3.1261.

8. Bhavani $C S$, Shah $H$, Dalal $A B$, et al. Novel and recurrent mutations in WISP3 and an atypical phenotype. Am J Med Genet A 2015; 167A:2481-4.

9. Sailani MR, Chappell J, Jingga I, et al. WISP3 mutation associated with pseudorheumatoid dysplasia. Cold Spring Harb Mol Case Stud 2018:4:a001990. doi: 10.1101/mcs.a001990.

10. Wickrematilake G. Progressive pseudorheumatoid dysplasia or IIA? Case Rep Rheumatol 2017; 2017:1609247. doi: $10.1155 / 2017 / 1609247$.

11. Garcia Segarra N, Mittaz L, Campos-Xavier AB, et al. The diagnostic challenge of progressive pseudorheumatoid dysplasia (PPRD): a review of clinical features, radiographic features, and WISP3 mutations in 63 affected individuals. Am I Med Genet C Semin Med Genet 2012; 160C:217-29. 